\title{
Medical Outcomes Study Physical Functioning Scale
}

National Cancer Institute

\section{Source}

National Cancer Institute. Medical Outcomes Study Physical Functioning Scale. NCI

Thesaurus. Code C110965.

A survey of multiple dimensions of support, representing the physical functioning scale. 